\title{
Un programa de Tratamiento de corte Cognitivo-Comportamental basado en las TIC para la prevención y el tratamiento de la depresión
}

\author{
Descripción del programa y datos preliminares
}

\author{
AdRIANA MIRA PASTOR \\ miraa@uji.es \\ CRistina Botella ARBona \\ botella@uji.es \\ ANTONIO RIERA LÓPEZ DEL AMO \\ ariera@uji.es \\ INÉS MORAGREGA BERgARA \\ moragreg@uji.es \\ CARLA SOLER ROVIRA \\ solerc@uji.es
}

\section{Resumen}

Introducción: En el año 2020 la depresión alcanzará el segundo lugar del ranking de los DALYs (Disability Adjusted Life Years), por ello su prevención es un punto central en el Pacto Europeo para la Salud Mental y el Bienestar. Actualmente, menos del $50 \%$ de las personas afectadas recibe el tratamiento correcto. El uso de internet facilita el acceso a los Tratamientos Basados en la Evidencia Además, es importante desarrollar programas de prevención. Nuestro grupo ha desarrollado un programa preventivo, de corte cognitivo comportamental, auto-aplicado a través de Internet. Sonreír es Divertido combina los procedimientos eficaces para el manejo del estrés, la regulación emocional, la capacidad de afrontamiento y la resiliencia. Metodología: La muestra está compuesta por nueve participantes varones desempleados. La media de edad es de 36-33 (SD = 10-759), con un rango de 22 a 50 años. Se utilizaron: Escala general de Gravedad e Interferencia de la Ansiedad y de la Depresión, Inventario de Depresión de Beck-Il y Escala Multidimensional de Estilos de Afrontamiento. Resultados: No hay diferencias estadísticamente significativas del pre al postratamiento, a excepción de la subescala de Aceptación del Brief Cope $(z=-2,27$; $p<, 05)$. En el resto de variables, las medias muestran una tendencia a la mejoría. Conclusión: Este programa puede ser útil como estrategia para la prevención, ya que, a pesar de que los participantes estaban pasando por una situación difícil, se observa una tendencia a la mejoría.

Palabras clave: terapia basada en Internet, prevención, depresión, capacidad de afrontamiento, regulación emocional. 


\section{Introduction}

In 2020, depression will reach the second place in the DALYS (Disability Adjusted Life Years), ranking, so prevention is a central point for the European Pact for Mental Health and Wellbeing. Currently, less than $50 \%$ of those people affected receive the correct treatment. Using Internet facilitates the access to evidence-based treatments. It is also important to develop prevention programs. Our group has ellaborated a cognitive-behavioural preventive program, self-applied through the Internet. Smiling is Fun combines effective procedures for stress management, emotional regulation, coping ability and resilience. Methodology: The sample consists of nine participants. They are all unemployed men, and their mean age is $36{ }^{\prime} 33$ (SD $\left.=10^{\prime} 759\right)$, with a range of 22-50 years. We used: Overall Anxiety Severity and Impairment Scale, Overall Depression Severity and Impairment Scale, Beck Depression Inventory II and Brief COPE. Results: There aren't statistically significant differences between pre and post-treatment, except for the Acceptance subscale of the Brief Cope $(z=-2,27, p<.05)$. For all other variables, means show a tendency to improve. Conclusion: This program can be useful as a prevention strategy considering that even though the participants were going through a difficult situation, there is a trend towards improvement.

Keywords: Internet-based therapy, prevention, depression, coping, emotional regulation.

\section{Introducción}

Recientemente ha surgido evidencia acerca de la existencia de un solapamiento de las estructuras latentes y las características clínicas de los trastornos de ansiedad y los trastornos del estado del ánimo, conformando ambos los denominados Trastornos Emocionales (TE) (Wilamowska et al., 2010). Estos trastornos dan lugar a una reducción de la calidad de vida de las personas convirtiéndose a menudo en trastornos crónicos y muy incapacitantes (Slade, Johnston, Oakley-Browne, Andrews y Whiteford, 2009). Los TE se han convertido en uno de los problemas más importantes para la salud a nivel mundial y su coste, tanto personal, como social (familia, amigos) y económico es muy elevado. La Organización Mundial de la Salud, estima que los problemas de salud mental suponen entre el $3 \%$ y $4 \%$ del producto nacional bruto de los Estados miembros de la Unión Europea (Gabriel y Liimatainen, 2000), y dentro de estos, la depresión y los trastornos de ansiedad son los más frecuentes, afectando a millones de personas cada año. En Europa, el 25-35 \% de las consultas en atención primaria tienen que ver con salud mental y más del $80 \%$ de estas son por depresión o ansiedad (WHO Iniciative Depression in Mental Health, 2010). Se sabe que el $25 \%$ de las personas padecerá depresión en algún momento de su vida y de acuerdo con la Organización Mundial de la Salud se espera que en el año 2020 alcance el segundo lugar del ranking de los DALYS (Disability Adjusted Life Years) (WHO Iniciative Depression in Mental Health, 2010), ya que se está produciendo un incremento exponencial en el número de afectados (niños, adultos, ancianos...). A este estado de cosas, cabe añadir la carencia de programas debidamente protocolizados, de amplio abordaje terapéutico, de fácil diseminación y las elevadas tasas coste-beneficio (Gaston, Abbott, Rapee y Neary, 2006). La depresión se asocia con un aumento de la mortalidad especialmente a través del suicidio. La investigación ha demostrado que el $90 \%$ de las personas que se 
suicidan tienen depresión u otro diagnóstico de trastorno mental o de abuso de sustancias (Moscicki, 2001). Por eso no es de extrañar que la prevención de la depresión y el suicidio se haya planteado como uno de los puntos centrales en el Pacto Europeo para la Salud Mental y el Bienestar (European Pact for Mental Health and Wellbeing, 2008). Estos datos subrayan la importancia de desarrollar estrategias y diseñar herramientas para identificar a las personas en riesgo, así como diseñar programas de prevención.

En las últimas décadas, los dos tipos de terapia que han acumulado evidencia sobre su eficacia para el tratamiento de la depresión son la terapia cognitivo comportamental (TCC) y la terapia interpersonal (TIP) (Antony y Stein, 2009; Nathan y Gorman, 2007; Norton y Price, 2007). Se trata de protocolos estandarizados y manualizados que se pueden aplicar en diversos contextos. Ambos tipos de terapia han demostrado ser eficaces, sin embargo, también presentan limitaciones. En primer lugar, menos del $50 \%$ de las personas con TE recibe el tratamiento correcto, ya sea por el coste económico, el tiempo requerido en su aplicación y/o la falta de profesionales bien entrenados (Andrade et al., 2003); a esto, además, se une el estigma que sigue existiendo por el hecho de ser tratado por un profesional de salud mental (Weissman et al., 1996). Todo esto hace que muchos pacientes no se decidan o tengan dificultades para buscar ayuda (Titov et al., 2011). La importancia del problema que suponen los TE y la necesidad urgente de resolverlo se está poniendo de manifiesto en la literatura científica. En noviembre de 2009 la prestigiosa revista Behavior Research and Therapy dedicó un número monográfico a este aspecto, en el que contribuyeron los investigadores más prestigiosos en el campo, como Rachman, Barlow, Clark, Craske, Salkovskis, Ehlers, Garety, Freeston, etc. Estos trabajos y otros muchos (Gunter y Whittal, 2010) ponen de relieve la necesidad de delimitar y superar las barreras a la diseminación de los TCC basados en la evidencia (TCCBE).

Un enfoque que facilita el acceso a los TCCBE es el uso de programas de tratamiento que utilizan como soporte Internet (ITCC). Los pacientes trabajan a partir de una guía de autoayuda adaptada para Internet y basada en protocolos de tratamiento basados en la evidencia. Se implica de forma sistemática a la persona en completar varias lecciones en línea que, por lo general, presentan la misma información y las técnicas que se utilizan en la Tcc cara a cara, a menudo con el apoyo de un clínico entrenado para ello (Titov et al., 2011).

Esta alternativa, focalizada en intervenciones de autoayuda a través de Internet ha demostrado ser una intervención eficaz y de bajo coste para los trastornos emocionales.

En España, nuestro grupo fue primero en todo el mundo en presentar un programa que utiliza Internet con estrategias de autoayuda y que puede ser totalmente autoaplicado (Botella et al., 2000). Este programa es Háblame y está dirigido al tratamiento del miedo a hablar en público. Los datos de un estudio de caso y de series de casos indican que Háblame es eficaz ( Botella et al., 2007). Este programa fue sometido a prueba en un estudio controlado y se comprobó que usado en formato autoaplicado resulta tan eficaz como administrado por un terapeuta, y que los cambios se mantienen al año de seguimiento (Botella et al., 2010). El segundo programa desarrollado por nuestro grupo fue Sin miedo (Botella et al., 2008), también es un programa de tratamiento autoaplicado para la fobia a animales pequeños (ratas, arañas y cucarachas). Diversos estudios han aportado datos que demuestran su eficacia (Baños, Quero, Botella y García-Palacios, 2007; Botella et al., 2008).

Otros autores han desarrollado programas de tratamiento que utilizan como soporte Internet obteniendo también buenos resultados. Especialmente, en el campo de los TE, destacan los trabajos realizados por los grupos de Isaac Marks y Judith Proudfoot en el Reino Unido (Marks, Cavannagh y Gega, 2007; McCrone, Marks, Mataix-Cols, Kenwright y McDonough, 2009) y por el grupo de Gavin Andrews y Nikolai Titov en Australia (Andrews y Titov, 2010; Titov, Andrews, Choi, Schwencke y Johnston, 2009). 
Las revisiones sistemáticas que se han realizado muestran que los tratamientos a través de Internet y los tratamientos computarizados son intervenciones eficaces (Cuijpers, Van Straten, Van Oppen y Andersson, 2008).

Además de seguir desarrollando programas de tratamiento que utilizan como soporte Internet, como los mencionados anteriormente, ayudando así a superar la barrera de la diseminación de los TCCBE, es importante desarrollar mejores estrategias y herramientas para detectar a las personas en riesgo, así como diseñar programas de prevención. Un desafío que en la actualidad resulta muy relevante es afrontar el tema de los TE desde esta perspectiva preventiva. Aunque existen tratamientos eficaces disponibles, los investigadores han defendido que cada vez son necesarios mayores esfuerzos para prevenir la aparición inicial de los TE. Con este propósito, se han desarrollado y evaluado diversos programas que han mostrado resultados de interés y las revisiones de meta-análisis muestran resultados positivos (Horowitz y Garber, 2006; Jané-Llopis, Hosman, Jenkins y Anderson, 2003; Cuijpers et al., 2008).

Como se ha señalado anteriormente, el uso de Internet está suponiendo un nuevo modo de dispensar TCCBE, y resulta también de gran ayuda en el caso de la prevención. Existen intervenciones de prevención autoaplicadas a través de Internet que han demostrado ser eficaces en la reducción de los síntomas de la depresión y ansiedad. Uno de estos programas de prevención que ha obtenido buenos resultados es el Internet-based stress managment (Andersson, Bergström, Carlbring y Lindefors, 2004). Recientemente, se ha desarrollado un nuevo programa de prevención autoaplicado a través de Internet (Van Straten, Cuijpers y Smits, 2007) que recibe el nombre de Dutch Internet-based PST intervention y está basado en la SelfExamination Therapy (SET). Esta intervención ha tenido éxito en la reducción de los síntomas de depresión, ansiedad y estrés relacionado con el trabajo en población adulta (Van Straten, Cuijpers y Smits, 2007). Otro programa preventivo, en este caso universal, también autoaplicado a través de Internet, es el MoodGYM, dirigido a jovenes, desarrollado por el grupo de Christensen en Australia, (Calear, Christensen, Mackinnon, Griffiths y O'Kearney, 2009; Sethi, Campbell y Ellis, 2010). Se han encontrado resultados significativos en la reducción de los síntomas depresivos (O'Kearney, Kang, Christensen y Griffiths, 2009). También en Australia el grupo de Klein ha desarrollado programas para la prevención de ansiedad obteniendo buenos resultados (Online Anxiety Prevention Program e-PASS) (Kenardy, McCafferty y Rosa, 2006).

Los diferentes resultados obtenidos en los estudios con estos programas aportan evidencia sobre la eficacia de los programas basados en Internet para la prevención de los TE (depresión y ansiedad) (Calear y Christensen, 2010).

Las intervenciones preventivas han tratado pues de evitar la aparición de la depresión y la ansiedad mediante la construcción de habilidades de afrontamiento y habilidades para el manejo del estrés, por lo que los programas preventivos que se han desarrollado en su mayoría tienen su fundamento en las estrategias cognitivo-conductuales. En la medida que se ha demostrado la eficacia de esta forma de terapia, se ha supuesto que estas habilidades y comportamientos podrían enseñarse antes de que la persona se deprima o desarrolle un trastorno de ansiedad, lo que en principio evitaría, o haría menos probable, la aparición de los mismos. De hecho, hay una estrecha relación entre el estrés, depresión y la capacidad de afrontamiento de la persona (Thompson et al., 2010). El afrontamiento inadecuado se ha asociado a altos niveles de estrés, síntomas de ansiedad y depresión, tanto en adolescentes (Marcks y Woods, 2005) como en adultos (Morillo, Belloch y García-Soriano, 2007). Además del afrontamiento inadecuado, las personas con trastornos emocionales utilizan estrategias de regulación emocional desadaptativas que contribuyen a la presencia de sus síntomas (Barlow et al., 2011).

En base a todo esto, y teniendo en consideración la alta prevalencia de los TE y el aumento exponencial del número de personas que pueden ver alterada notablemente su calidad de vida, su bienestar y su funcionamiento personal, resulta necesario desarrollar herramientas 
capaces de detectar las personas en riesgo y programas de prevención eficaces. Además, teniendo en cuenta los avances que ya se han demostrado en el uso de Internet para la diseminación de los mismos, parece también necesario intentar combinar todas estas estrategias. Nuestro grupo ha desarrollado un programa de intervención de corte cognitivo-comportamental, preventivo, interactivo y totalmente autoaplicado a través de Internet (Sonreír es divertido). La hipótesis fundamental es que este programa permitirá a la persona aprender y practicar formas adaptativas para afrontar el estrés y los problemas cotidianos y, de este modo, resultará de utilidad para prevenir la aparición de la depresión. En el presente trabajo vamos a describir en qué consiste Sonreír es divertido y presentar los datos preliminares pre-post intervención de los primeros 9 participantes que han utilizado el programa.

\section{Método}

\section{Participantes}

La muestra está compuesta por nueve participantes voluntarios. Todos son varones desempleados. La media de edad es de 36-33 (SD = 10-759), con un rango de 22 a 50 años. En cuanto al estado civil, 4 de los participantes estaban solteros, 4 casados o en pareja y uno divorciado. Por lo que respecta a su nivel de formación académica, 7 de ellos tienen estudios superiores, 1 ha cursado secundaria y el otro tiene un nivel de estudios básicos.

Criterios de inclusión/exclusión: Estar en situación de desempleo fue una condición necesaria para entrar en el estudio; además, los participantes tenían que estar pasando una situación vital difícil que pudiera comportar problemas económicos, cargas familiares, dificultades en la vida cotidiana y con ello un aumento de estrés. La edad tenía que estar comprendida entre los 18 y 65 años, tenían que estar dispuestos a participar en el estudio dando su consentimiento informado, saber utilizar el ordenador y tener acceso a Internet con el fin de acceder al programa. Los criterios de exclusión incluyeron historia personal de depresión (BDI-II $\geq 19$ ) y/o psicosis, historia de depresión y/o psicosis en cualquier familiar de primer grado (padres o hijos de los participantes), la ingesta regular de drogas recreativas (cannabis, etc.) y padecer una enfermedad grave que pudiera interferir con la realización del programa.

\section{Instrumentos}

Los instrumentos y medidas utilizados siguen a continuación:

\section{1) Entrevista diagnóstica}

Entrevista Neuropsiquiátrica Internacional (Mini-International Neuropsychiatric Interview, MINI; Lecrubier et al. 1997). Esta es una breve entrevista estructurada de diagnóstico psiquiátrico que evalúa los diagnósticos clave de DSM-IV y CIE-10 (trastornos de ansiedad, del estado del ánimo, psicosis, abuso y dependencia de sustancias...). Ha sido traducida y validada en español (Sheehan et al. 1997).

2) Cuestionarios y escalas de autoinforme

Escala general de gravedad e interferencia de la ansiedad (Overall Anxiety Severity and Impairment Scale, OASIS; Norman et al., 2006). Es una medida continua de autoinforme confor- 
mada por 5 ítems que se codifican de 0 a 4 y se suman para obtener una puntuación total. Esta escala se puede utilizar para evaluar la gravedad y el deterioro asociado a cualquier trastorno de ansiedad, trastornos múltiples de ansiedad o síntomas subliminales cuando no se cumplen los criterios de ningún trastorno de ansiedad particular. Un análisis psicométrico de la escala OASIS determinó una buena consistencia interna (alfa de Cronbach $=0,80$ ), fiabilidad test-retest ( $k=5,82)$ y validez convergente (Norman et al., 2006) para esta escala.

Escala general de gravedad e interferencia de la depresión (Overall Depression Severity and Impairment Scale, ODSIS; Norman et al., 2006). Al igual que el OASIS, el ODSIS es una medida de autoinforme conformada por 5 ítems que se codifican de 0 a 4 y se suman para obtener una puntuación total. En este caso, se evalúan experiencias relacionadas con la depresión. Hasta el momento, no se han publicado estudios que analicen las propiedades psicométricas de esta escala. Norman et al., (2006) recomiendan utilizarla e interpretarla de la misma forma que el OASIS.

Inventario Depresión de Beck-Segunda edición (Beck Depression Inventory-Second Edition, BDI-Il; Beck, Steer y Brown, 1996. Validada en población española por Sanz, Navarro y Vázquez, 2003). Este instrumento esta constituido por 21 grupos de afirmaciones que evalúan síntomas y cogniciones relacionadas con la depresión. El participante debe responder de acuerdo con la afirmación que mejor describa el mondo en el que se ha sentido durante las últimas dos semanas. La versión española de este instrumento ha mostrado una consistencia interna elevada (alfa de Cronbach de 0,87) para la población general (Sanz, Perdigón y Vázquez, 2003) y para la población clínica (alfa de Cronbach de 0,89).

Escala Multidimensional de Estilos de Afrontamiento (abreviado) (Brief COPE, Carver, 1997). El cuestionario está compuesto por 14 dimensiones, cada una con dos ítems, y permite evaluar los diversos estilos de afrontamiento. El cuestionario ha mostrado una buena consistencia interna con un alfa de Cronbach entre 0,60 y 0,90 (Crespo y Cruzado, 1997).

\section{Diseño}

El presente trabajo es un estudio piloto donde se presentan los datos pre- y postratamiento de una serie de casos (9 participantes).

\section{Descripción del programa}

Sonreír es divertido es un programa de tratamiento de corte cognitivo-comportamental, que se sustenta en las TIC y tiene como soporte la aplicación de Internet. La persona puede realizar los módulos desde casa, de manera totalmente autoapilcada, recorriendo el sistema y realizando las distintas tareas a su ritmo y en los horarios que considere más convenientes.

Tiene un doble objetivo: Por una parte pretende ser un programa de prevención para ayudar a aquellas personas en alto riesgo, es decir, sometidas a situaciones difíciles y/o estresantes ofreciéndoles técnicas y estrategias para afrontar dichas situaciones de manera eficaz; Por otra, tratar a personas que ya presenten algún trastorno emocional o trastorno adaptativo de gravedad leve o moderada.

Está basado en la perspectiva transdiagnóstica (Brown y Rosellini, 2011; Dudley, Kuyken y Padesky, 2011; McLaughlin y Nolen-Hoeksema, 2011; Mansell, 2012) en concreto, en algunos los componentes del Unified Protocol del grupo de Barlow: Motivación, Psicoeducación y Terapia cognitiva (Barlow et al., 2011). Además, el programa incorpora técnicas para prevenir y tratar el estrés y la depresión, tales como la activación comportamental. A esto se suman 
estrategias para fomentar y mejorar el estado de ánimo positivo. El programa consta pues de 5 componentes terapéuticos: Motivación, Psicoeducación, Terapia Cognitiva, Activación Comportamental y Psicología positiva.

Trata de combinar los procedimientos más eficaces existentes para el manejo del estrés psicológico y el bajo estado de ánimo con estrategias para promocionar la regulación emocional, la capacidad de afrontamiento y la resiliencia. Al diseñarlo se han tenido en cuenta todos los avances potenciando de forma importante los módulos centrados en la promoción del afecto positivo así como subrayando notablemente la importancia y los beneficios de estar activo y de mantenerse implicado en la vida y en los propios valores y metas.

El programa empieza con el modulo Home donde se explica en qué consiste, cuál es el objetivo, quién se puede beneficiar de su realización y quiénes son los profesionales que están detrás. Se utilizan una serie de elementos multimedia (textos, viñetas, imágenes, vídeos...) para explicar todo esto subrayando de forma especial los ejemplos de personas que se pueden beneficiar del programa (véase figura 1).

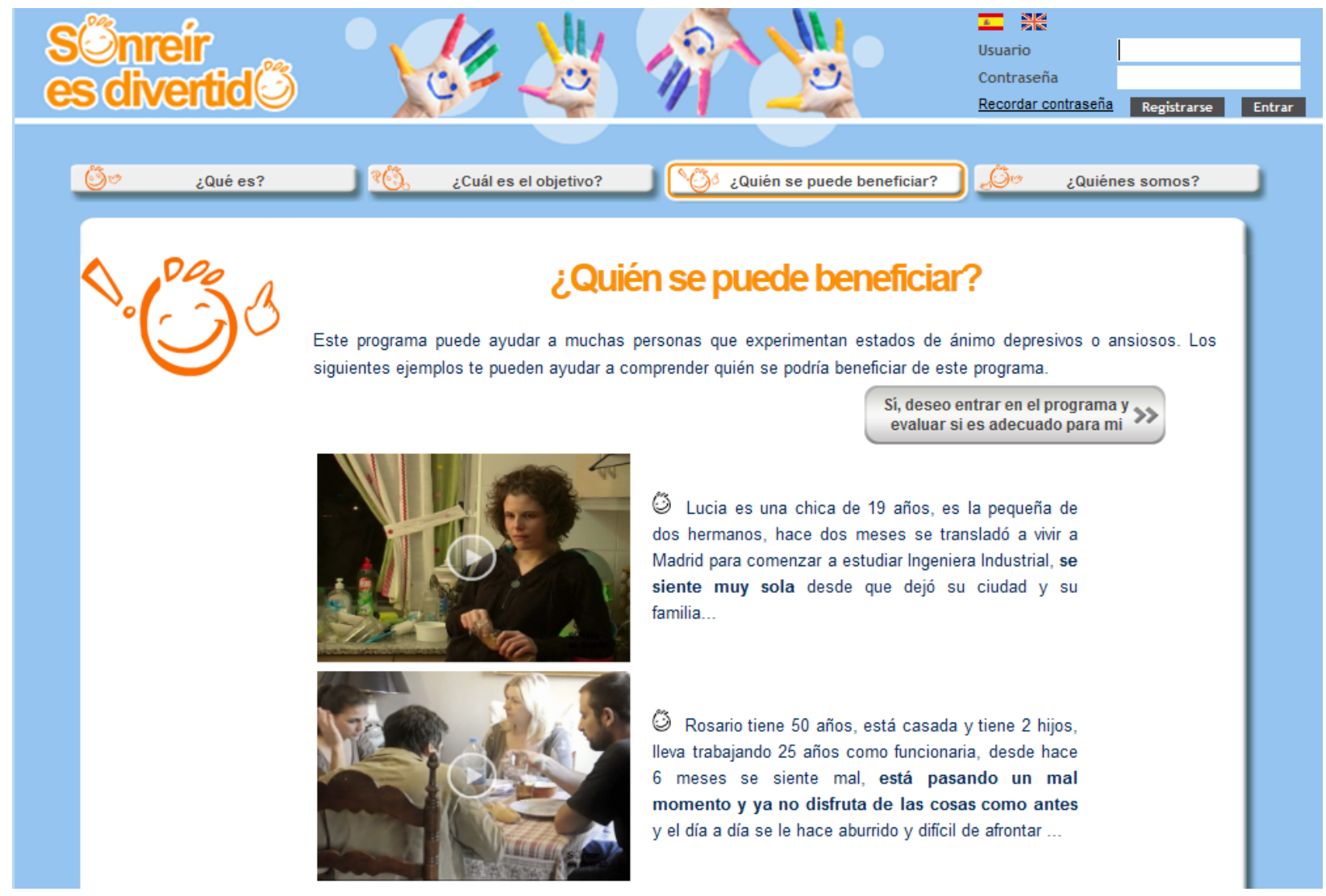

Figura 1. Módulo Home

Después del modulo Home sigue el módulo de Bienvenida, donde se proporciona al usuario una información acerca del contenido de los módulos que se incluyen en el programa y recomendaciones importantes para que se pueda beneficiar máximamente de este.

El programa realiza una evaluación pretratamiento, una evaluación a lo largo de todo el programa (después de cada uno de los módulos), una evaluación postratamiento, y evaluaciones de seguimiento (a los 3, 6 y 12 meses) integradas dentro del propio sistema web. 
Una vez realizada la evaluación pretratamiento, siguen los 8 módulos que conforman Sonreír es divertido: M1. Motivación para el cambio; M2. Entendiendo los problemas emocionales; M3. Aprendiendo a ponerse en marcha; M4. Aprendiendo a ser flexibles; M5. Aprendiendo a disfrutar: La importancia de las experiencias positivas; M6. Aprendiendo a vivir: la importancia de los valores y las metas; M7. Viviendo y aprendiendo; M8. ¿Y a partir de ahora qué...? Todos ellos estan orientados a ayudar a aprender distintas técnicas psicológicas que permitan a la persona conocer formas adaptativas de afrontar el estrés cotidiano y los problemas.

El diseño se ha orientado a optimizar la comprensión del contenido de los módulos, enriqueciéndolo con distintos elementos multimedia (viñetas, vídeos, audios...) según la conveniencia de cada momento, para ayudar al usuario a asimilar de la forma más fácil las distintas técnicas psicológicas que se presentan en el programa.

Los aspectos fundamentales de Sonreír es divertido trabajados en cada uno de los módulos están basados en técnicas que han demostrado su eficacia y se ajusta a las recomendaciones de las guías sobre buenas prácticas clínicas de las asociaciones internacionales de psicología como la American Psychological Association (www.apa.org) y el National Institute for Health and Clinical Excellence (www.nice.org.uk).

Además de los 8 módulos, Sonreír es divertido ofrece una serie de herramientas que acompañan al usuario a lo largo de todo el proceso (herramientas transversales):

El diario de actividad es una herramienta diseñada para que la persona preste atención a las actividades que realiza cotidianamente, a qué dedica el tiempo y cómo influye esto en su estado de ánimo (véase figura 2).

El calendario es una herramienta que proporcionará información a la persona acerca de sus avances a lo largo del programa. Le permitirá saber en qué lugar se encuentra, lo que te falta para terminar y le dará feedback sobre las tareas que ya ha realizado y las que aún tiene pendientes. (véase figura 3 ).

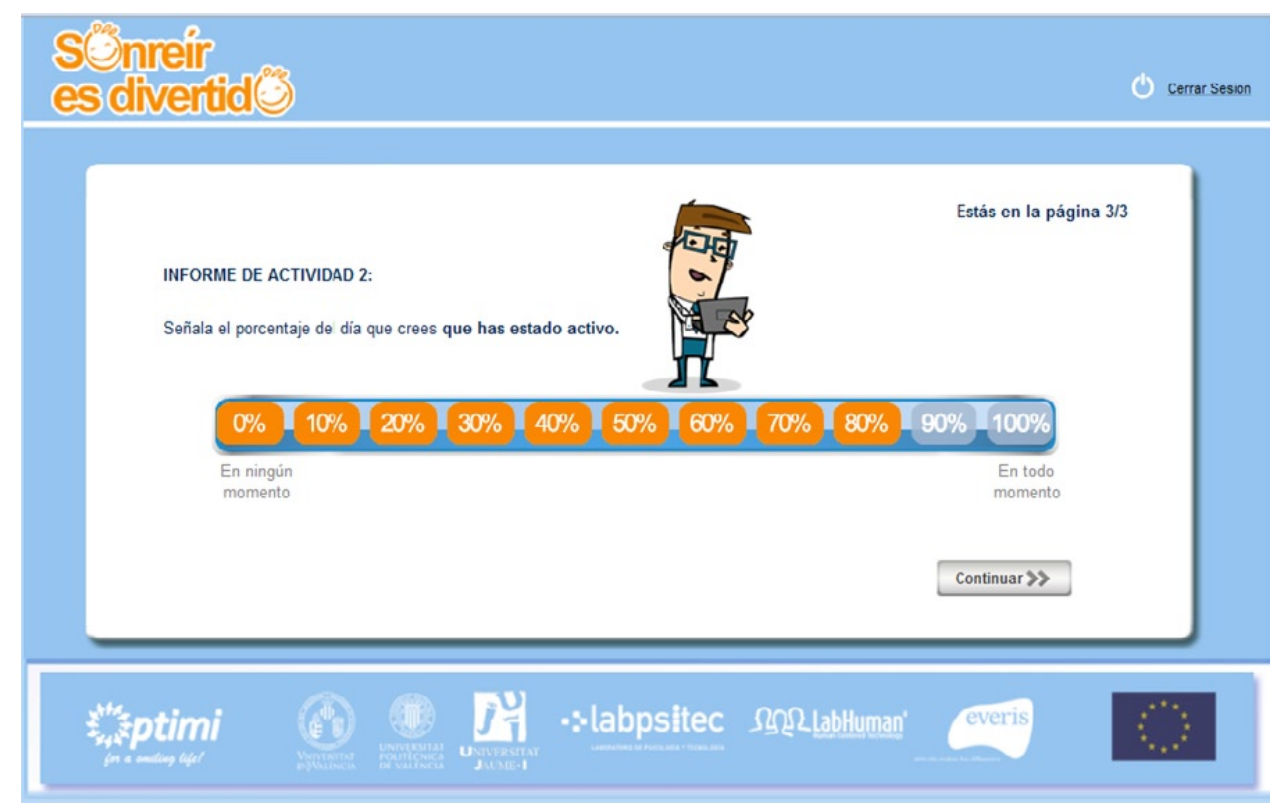

Figura 2. Diario de Actividad 


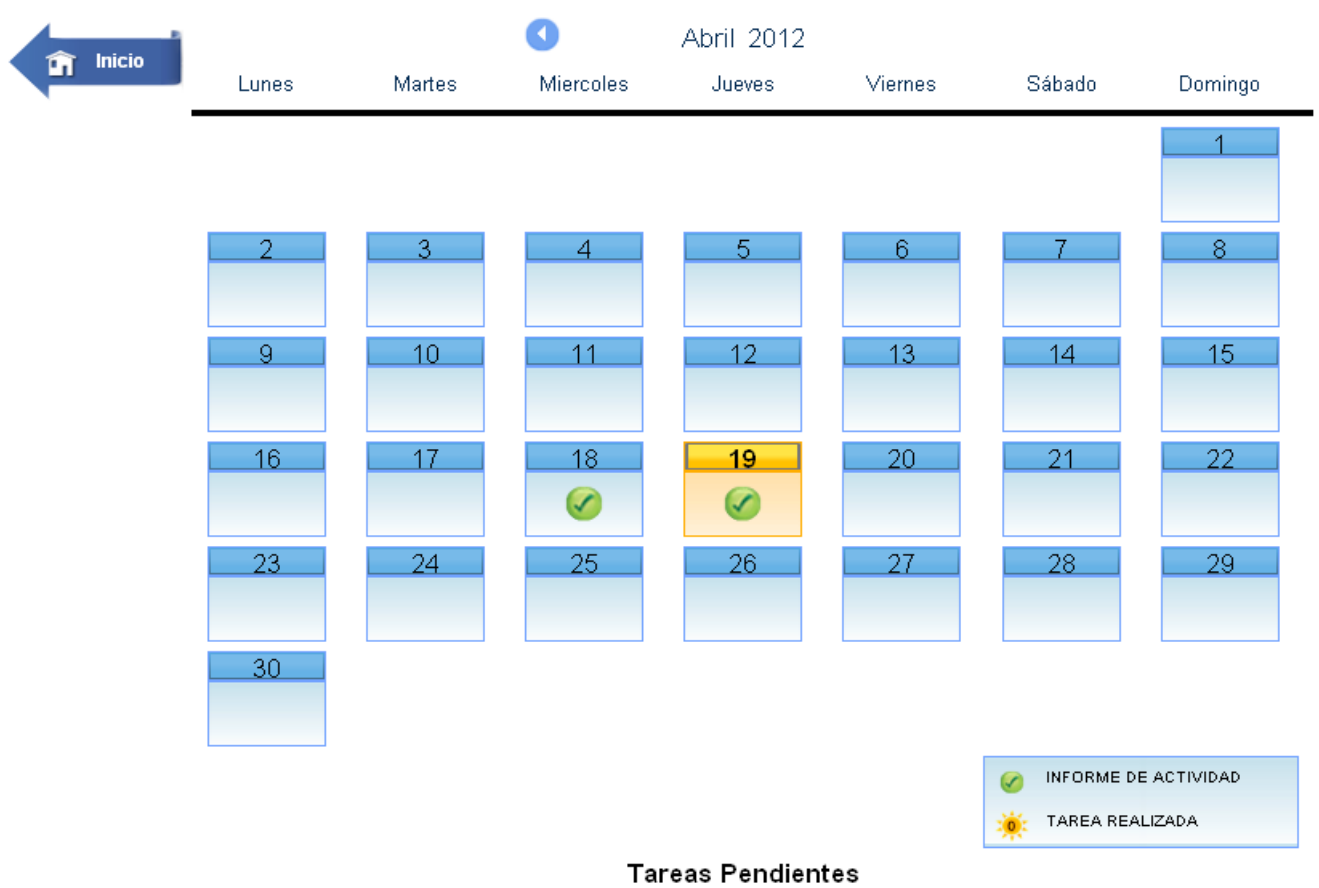

Figura 3. Calendario

¿Cómo estoy? es una herramienta que ofrece feedback a la persona de forma gráfica sobre su evolución a lo largo del programa tanto de su nivel de actividad, de su malestar emocional (ansiedad y tristeza) como de la intensidad de su emocionalidad positiva (activo, ilusionado, con energía, con vitalidad, etc.) y negativa (disgustado, con miedo, estresado, tenso, malhumorado, etc.).

\section{Procedimiento}

Primero se hizo un llamamiento a través de carteles anunciados en la Universitat Jaume I, a través de Internet y de diferentes medios de comunicación (radio, prensa...). Nos pusimos en contacto telefónicamente con los interesados para delimitar la presencia o ausencia de los criterios de exclusión más importantes. A los participantes que pasaron dicho screening telefónico se dio cita para una entrevista inicial. Los participantes acudían al Laboratorio de Psicología y Tecnología, allí un investigador del equipo explicaba lo que implicaba la participación en el estudio y el participante firmaba su consentimiento informado. Posteriormente se evaluaba el posible diagnóstico de depresión mayor con la Entrevista Neuropsiquiátrica Internacional (MINI) y con la puntuación del participante en el Inventario de Depresión II de Beck, pues puntuar más de 19 era uno de los criterios de exclusión del estudio. Además, los participantes completaban una serie de cuestionarios de autoinforme. Finalmente, los que cumplieron con los criterios de inclusión pasaron a formar parte del estudio.

Los participantes recibían una llamada de apoyo semanal por parte del clínico a lo largo de todo el proceso de realización del programa (2 minutos). 


\section{Resultados}

Hemos realizado un análisis no paramétrico para dos muestras relacionadas con la prueba Wilcoxon, mediante el SPSS 15.0. En la tabla 1 se muestran las medias, desviaciones típacas y la significación estadística de las variables analizadas. Podemos observar estabilidad en todas las variables clínicas, sin diferencias estadísticamente significativas del pretratamiento al postratamiento, a excepción de la subescala de Aceptación del Brief Cope, en la que sí que si se produce una mejora estadísticamente significativa del pre al postratamiento $(z=-2,27$; $p<, 05)$. Aunque no haya diferencias estadísticamente significativas en el resto de variables, podemos observar que las medias muestran una tendencia a la mejoría.

Tabla 1

Prueba no paramétrica de los signos de Wilcoxon para la comparación entre las variables analizadas en el pretratamiento $y$ en el postratamiento

\begin{tabular}{lcccccc}
\hline \multirow{2}{*}{ Variable } & \multicolumn{2}{c}{ Pre } & \multicolumn{2}{c}{ Post } & Z & p \\
\cline { 2 - 5 } & Media & DT & Media & DT & & \\
\hline OASIS & 2,22 & 2,49 & 1,67 & 2,06 &,- 85 &, 39 \\
\hline ODSIS & 1,33 & 2,65 &, 78 & 1,64 &,- 82 &, 41 \\
\hline BDI-II & 5,44 & 6,44 & 4,89 & 5,97 &,- 90 &, 37 \\
\hline Cope: Afront. & 6,11 & 1,36 & 6,22 & 1,20 &,- 38 &, 70 \\
\hline Cope: A. Emo. & 4,33 & 2,12 & 5,11 & 1,76 & $-1,47$ &, 14 \\
\hline Cope: A. Soci. & 4,56 & 1,67 & 5,11 & 1,83 & $-1,52$ &, 13 \\
\hline Cope: Acepta. & 5,11 & 1,27 & 6,00 & 1,12 & $-2,27$ &, 02 \\
\hline Cope: Negaci. & 2,11 &, 33 & 2,33 &, 707 &,- 82 &, 41 \\
\hline
\end{tabular}

Nota. Pre = Pretratamiento; Post= Post- tratamiento; Cope: Afront. Subescala Afrontamiento Activo del Brief Cope; Cope: A. Emo. =Subescala Apoyo Emocional del Brief Cope; Cope: A. Soci.= Subescala Apoyo Social del Brief Cope; Cope: Acepta.=Subescala Aceptación del Brief Cope; Cope: Negaci. = Subescala Negación del Brief Cope.

\section{Discusión y conclusiones}

Como se ha señalado en la introducción, este programa (Sonreír es divertido), autoaplicado a través de Internet, ha considerado los elementos prácticos y conceptuales que están en la base de los principales programas de prevención en este ámbito desarrollados en el mundo, que, en su mayoría, emplean estrategias y técnicas desarrolladas desde los enfoques cognitivocomportamentales para el tratamiento de la depresión (Collins y Dozois, 2008). Además, cabe subrayar el interés de este estudio en incorporar otras técnicas para prevenir y tratar el estrés y la depresión, tales como la activación comportamental, ya propuesta en el pasado por el propio Beck (Beck, 1976), enseñando a la persona la importancia de adquirir un nivel adecuado de actividad e implicación con la vida, mostrando las consecuencias del abandono de actividades, así 
como los beneficios del ejercicio físico (Ekers, Richards, McMillan, Bland y Gilbod, 2011; MotaPereira, Silverio, Carvalho, Ribeiro, Fonte y Ramos, 2011). A esto se suma la incorporación de estrategias para fomentar y mejorar el afecto positivo. Cada vez hay más soporte en la literatura que avala que las emociones positivas promueven un pensamiento flexible y creativo y juegan un papel fundamental en la construcción de fortalezas psicológicas, intelectuales y recursos sociales que pueden ser de utilidad en situaciones futuras difíciles (Algoe y Fredrickson, 2011; Catalino y Fredrickson, 2011; McMahan y Renken, 2011; Wood, Froh y Geraghty, 2011).

Los resultados obtenidos en la presente investigación muestran que todos los participantes presentaban niveles bajos de ansiedad en el pretratamiento (evaluada mediante en OASIS) y estos niveles de ansiedad se han mantenido estables, sin diferencias estadísticamente significativas del pre al postratamiento. En cuanto a la sintomatología depresiva (evaluada mediante el ODSIS y el BDI-II), las puntaciones también se han mantenido estables, mostrando niveles bajos de sintomatología depresiva en el pretratamiento y en el postratamiento, sin diferencias estadisticaemnte significativas.

Cabe señalar que las medias en el post tratamiento, tanto en el OASIS, en el ODSIS como en el BDI-II muestran una tendencia a la mejoria.

Todos estos resultados ponen de manifiesta la posible utilidad de este programa como estrategia para la prevención de la depresión, ya que, a pesar de que los participantes estaban pasando por una situación vital difícil como supone estar en paro, que se mantenía en el tiempo, por lo que a medida que el tiempo transcurría la situación empeoraba; y a demás referían tener cargas familiares o dificultades en la vida cotidiana (enfermedad crónica de un familiar, conflictos familiares etc.) y con ello un aumento de estrés, los síntomas de depresión y ansiedad se mantuvieron estables en la mayoría de los casos e incluso mejoraron en algunos.

Por lo que respecta a las estrategias de afrontamiento positivas utilizadas por los participantes, vemos que las medias del post tratamiento reflejan una tendencia a la mejoría, por lo que los participantes han mantenido o han fortalecido diferentes estrategias adaptativas de afrontamiento como son iniciar acciones directas e incrementar los propios esfuerzos para eliminar o reducir al estresor (afrontamiento activo), conseguir apoyo emocional de simpatía y de comprensión (apoyo emocional), procurar ayuda, consejo, información a personas que son competentes acerca de lo que deben hacer (apoyo social) y aceptar el hecho de lo que está ocurriendo (aceptación), existiendo en esta última variable clínica diferencias estadísticamente significativas del pre al postratamiento.

Teniendo en cuenta todo esto, cabe afirmar que este programa de tratamiento autoaplicado a lo largo de 8 semanas ha logrado uno de sus objetivos fundamental, a saber, conseguir proveer a las personas de las herramientas y habilidades necesarias para fortalecer y/o mejorar sus estrategias de afrontamiento. Estos resultados están en concordancia con los datos de la literatura que avalan la estrecha relación entre el estrés, depresión y la capacidad de afrontamiento de la persona (Thompson et al., 2010), estando asociado el afrontamiento inadecuado con altos niveles de estrés, síntomas de ansiedad y depresión, tanto en adolescentes (Jaser et al., 2005; Marcks y Woods, 2005) como en adultos (Morillo, Belloch y García-Soriano, 2007; Sarin, Abela, y Auerbach, 2005). Enseñar formas adaptativas de afrontamiento se asocia pues con un mayor bienestar, tanto físico como psicológico (Hong, 2007).

Vemos pues que se ha producido lo esperado en la evolución de las diferentes variables clínicas del pre al postratamiento. Al tratarse de un programa de prevención, no se busca necesariamente una mejoría en el postratamiento, sino que el programa les proteja de la situación vital difícil que están viviendo y esto se refleje, tal y como sucede en los resultados del presente estudio, en una estabilidad en su sintomatología depresiva y ansiosa y en el mantenimiento, refuerzo y/o mejora de estrategias adaptativas para afrontar la situación vital complicada que está aconteciendo. Es importante subrayar que la muestra corresponde a población no clínica pero 
que está afrontando acontecimientos vitales estresantes (desempleo, desempleo de familiares, enfermedad crónica de un familiar, cargas familiares, conflictos familiares...), por lo que entendemos resulta esperanzador que, a pesar de que dichos acontecimientos continúan presentes, en general se observa una tendencia a la estabilidad o a la mejoría. No existen diferencias estadísticamente significativas del pretratamiento al postratamiento tal y como muestran los resultados del análisis no paramétrico para dos muestras relacionadas con la prueba Wilcoxon, a excepción de la subescala de Aceptación del Brief Cope $(z=-2,27 ; p<0,05)$. Es importante recalcar que en las medias del postratamiento en el resto de variables clínicas se observa una tendencia hacía la mejoría.

Sin embargo, este estudio tiene limitaciones. La limitación fundamental es que es un estudio preliminar basado en series de casos y habrá esperar a tener resultados más consistentes a partir de un estudio controlado. Otra de las limitaciones de este estudio es que no tenemos datos de los seguimientos, por lo que no podemos saber si el programa protege también a los participantes a largo plazo, ni saber si la tendencia a la mejoría que se observa en las medias de las diferentes variables clínicas llegará al final a tener significación como sucede en el caso de la Aceptación. Además, somos conscientes de que lo que se presenta son los datos preliminares de 9 participantes y esto, claramente, impide realizar generalizaciones. Por ello, en estos momentos estamos llevando a cabo un estudio controlado que esperamos nos permita aportar conclusiones más firmes.

Somos también conscientes de la necesidad de analizar y revisar cualquier tipo de aportación para poder avanzar en los planteamientos de la psicología basada en la evidencia y poder así delimitar programas de prevención eficaces autoaplicado a través de Internet para los trastornos emocionales.

\section{Referencias bibliográficas}

Algoe, S. B. \& Fredrickson, B. L. (2011). Emotional Fitness and the movement of affective science from lab to field. American Psychologist, 66(1), 35-42.

Andersson, G., Bergström, J., Holländer, F., Carlbring, P., Kaldo, V. \& Ekselius, L. (2005). Internet-based self-help for depression: randomised controlled trial. British Journal of Psychiatry, 187, 456-461.

Andrade, L., Caraveo-Anduaga, J. J., Berglund, P., Bijl, R. V., De Graaf, R., Vollebergh, W., ... Wittchen, H. U. (2003). The epidemiology of major depressive episodes: results from the International Consortium of Psychiatric Epidemiology (ICPE) Surveys. International Journal of Methods in Psychiatric Research, 12, 3-21.

Andrews, G. \& Titov, N. (2010). Is Internet treatment ready for prime time? Medical Journal of Australia, 192 (11), 45-47.

Antony, M. M. \& Stein, M. B. (2009). Oxford handbook of anxiety and related disorders. New York, NY, US: Oxford University Press.

Barlow, D. H., Ellard, K. K., Fairholme, C. P., Farchione, T. J., Boisseau, C. L. Allen, L. B. \& Ehrenreich-May, J. (2011). The unified protocol for transdiagnostic treatment of emotional disorders: Client workbook. New York, NY: Oxford University Press.

Beck, A. T. (1976). Cognitive therapy and the emotional disorders. New York: International Universities Press.

Baños, R. M., Quero, S., Botella, C. \& García-Palacios, A. (2007). Internet \& los tratamientos psicológicos: dos programas de auto-ayuda para fobias específicas Cuadernos de Medicina Psicosomática \& Psiquiatría de Enlace. 
Botella, C., Baños, R. M., Guillén, V., Perpiñá, C., Alcañiz, M. \& Pons, A. ( 2000). Telepsychology: Public Speaking Fear Treatment on The Internet. CyberPsychology \& Behavior, 3, 959986.

Botella, C., Gallego, M. J., García-Palacios, A., Guillen, V., Baños, R. M., Quero, S. \& Alcañiz, M. (2010). An Internet-Based Self-Help Treatment for Fear of Public Speaking: A Controlled Trial. Cyberpsychology Behavior, and Social Networking, 13, 407-421.

Botella, C., Guillén, V., García-Palacios, A., Gallego, M. J., Baños, R. M. \& Alcañiz, M. (2007). Telepsychology and self-help: The treatment of fear of public speaking. Cognitive and Behavioral Practice, 14, 46-57.

Botella, C., Quero, S., Baños, R. M., García-Palacios, A., Bretón-López, J. Alcañiz, M. \& Fabregat, S. (2008). Telepsychology and Self-Help: The treatment of phobias using the Internet. CyberPsychology \& Behavior 11, 659-664.

Brown, T. A. \& Rosellini, A. J (2011) The Direct and Interactive Effects of Neuroticism and Life Stress on the Severity and Longitudinal Course of Depressive Symptoms. Journal of Abnormal Psychology, 120(4), 844-856.

Bulacio, J. M., Vieyra, M. C., Alvarez Daneri, C. Benatuil, D. \& Mongiello, E. (2004). Índice de Calidad de Vida: Validación de una muestra Argentina. ALCMEON, 11, 333-336.

Calear, A. L., Christensen, H., Mackinnon, A., Griffiths, K. M. \& O'Kearney, R. (2009). The YouthMood project: A cluster randomized controlled trial of an online cognitive-behavioral program with adolescents. Journal of Consulting and Clinical Psychology, 77(6), 1021-1032.

Catalino, L. I. \& Fredrickson, B. L. (2011). Tuesdays in the lives of flourishers: The role of positive emotional reactivity in optimal mental health. Emotion, 11, 938-950.

Collins, K. A. \& Dozois, D. (2008). What are the active ingredients in preventative interventions for depression? Clinical Psychology: Sciencie and Practice, 15, 313-330.

Crespo, M. \& Cruzado, J. A. (1997). La evaluación del afrontamiento: adaptación española del cuestionario COPE con una muestra de estudiantes universitarios, 23, 797-830.

Cuijpers, P., Van Straten, A., Van Oppen, P. \& Andersson, G. (2008). Are psychological and pharmacological interventions equally effective in the treatment of adult depressive disorders? A meta-analysis of comparative studies. Journal of Clinical Psychiatry, 69, 1675-1685.

Dudley, R., Kuyken, W., Padesky, C. A. (2011). Collaborative Case Conceptualization: Working Effectively with Clients in Cognitive-Behavioral Therapy. Guilford Press.

Ekers, D., Richards, D., McMillan, D., Bland, M. \& Gilbody, S. (2011). Behavioural activation delivered by the non-specialist: phase II randomised controlled trial. British Journal of Psychiatry, 198 (1): 66-72.

European Pact for Mental Health and Wellbeing, (2008). EU high level conference. Tgether for metal health and wellbeig. Brussels.

Gabriel, P. \& Liimatainen, M. R. (2000). Mental health in the workplace. Geneva, InternationalLabourOffice.

Gaston, J. E., Abbott, M. J., Rapee, R. M. \& Neary, S. A. (2006). Do empirically supported treatments generalize to private practice? A benchmark study of a cognitive-behavioural group treatment programme for social phobia. British Journal of Clinical Psycholgy, 45, 33-48.

Gunter, R. W. \& Whittal, M. L. (2010). Dissemination of cognitive-behavioral treatments for anxiety disorders: Overcoming barriers and improving patients access. Clinical Psychology Review, 30, 194-202.

Hong, R. Y. (2007). Worry and rumination: differential associations with anxious and depressive symptoms and coping behavior. Behaviour Research and Therapy, 45, 277-290.

Horowitz, J. L. \& Garber, J. (2006). The prevention of depressive symptoms in children and adolescents: A meta-analytic review. Journal of Consulting and Clinical Psychology, 17, 401415. 
Jané-Llopis, E., Hosman, C., Jenkins, R. \& Anderson, P. (2003). Predictors of efficacy in depression prevention programmes: Meta-analysis. British Journal of Psychiatry, 183, 384-397.

Jaser, S. S., Langrock, A. M., Keller, G., Merchant, M. J., Benson, M. A., Reeslund, K, ... Compas B. E. (2005). Coping with the stress of parental depression II: adolescent and parent reports of coping and adjustment. Journal of Clinical Child and Adolescent Psychology, 34, 193-205.

Kenardy, J., McCafferty, K. \& Rosa, V. (2006). Internet-delivered indicated prevention for anxiety disorders: Six-month follow-up. Clinical Psychologist, 10, 39-42.

Lecrubier, Y., Sheehan, D., Weiller, E., Amorim, P., Bonora, I., Sheehan, K. \& Dunbar, G. (1997). The Mini international neuropsychiatric interview (M.I.N.I.), a short diagnostic interview: Reliability and validity according to the CIDI. Eur Psychiatry, 12, 232-241.

McCrone, P., Marks, M., Mataix-Cols, D., Kenwright, M. \& McDonough, M. (2009). Computer-aided self-exposure therapy for phobia/ panic disorder: A pilot economic evaluation. Cognitive Behaviour Therapy, 38 (2), 91-99.

McLaughlin, K. A. \& Nolen-Hoeksema, S. (2011) Rumination as a transdiagnostic factor in depression and anxiety. Behav Res Ther 49(3),186-93.

McMahan, E. A. \& Dehart Renken, M. (2011). Eudaimonic conceptions of well-being, meaning in life, and self-reported well-being: Initial test of a mediational model. Personality and Individual Differences, 51, 589-94.

Mansell, W. (2012). The Transdiagnostic Approach. En Dryden, W. (ed.), CBT Approaches to Counselling and Psychotherapy. Sage.

Marks, I. M., Cavannagh, K. \& Gega, L. (2007). Hands-on help: Computer-aided psychotherapy. Hove and New York: Psychology Press.

Marks, I. M., Mataix-Cols, D. Kenwright, M. Cameron, R. Hirsch, S. \& Gega, L. (2003). Pragmatic evaluation of computer-aided self-help for anxiety and depression. The British Journal of Psychiatry, 183, 57-65.

Marcks, B. A. \& Woods, D. W. (2005). A comparison of thought suppression to a acceptancebased technique in the management of personal intrusive thoughts: a controlled evaluation. Behaviour Research and Therapy, 43, 433-445.

Morillo, C., Belloch, A. \& García-Soriano, G. (2007). Clinical obsessions in obsessive compulsive patients and obsession-relevant intrusive thoughts in non-clinical, depressed and anxious subjects: where are the differences? Behaviour Research and Therapy, 45, 1319-1333.

Moscicki, E. K. (2001). Epidemiology of completed and attempted suicide: toward a framework for prevention. Clinical Neuroscience Research, 1, 310-23.

Mota-Pereira, J., Carvalho, S., Silverio, J., Fonte, D., Pizarro, A., Teixeira, J., ... Ramos, J. (2011). Moderate physical exercise and quality of life in patients with treatment-resistant major depressive disorder. J Psychiatr Res, 45 (12), 1657-1659.

Nathan, P. E. \& Gorman, J. M. (2007). A guide to treatments that work. Third Edition. New York, NY, US: Oxford University Press.

Norman, S. B., Cissell, S. H., Means-Christensen, A. J. \& Stein, M. B. (2006). Development and validation of an Overall Anxiety Severity And Impairment Scale (OASIS). Depres Anxiety, 23, 245-249.

Norton, P. J. \& Price, E. C. (2007). A meta-analytic review of adult cognitivebehavioral treatment outcome across the anxiety disorders. Journal of Nervous and Mental Disease, 195, 521531.

O'Kearney, R., Gibson, M., Christensen, H. \& Griffiths, K. M. (2006). Effects of a cognitive-behavioural internet program on depression, vulnerability to depression and stigma in adolescent males: a school-based controlled trial. Cognitive Behavioural Therapy, 35, 43-54. 
Sandín, B., Chorot, P., Lostao, L., Joiner, T. E., Santed, M. A. \& Valiente, R. M. (1999). Escalas Panas de Afecto Positivo \& Negativo: Validación Factorial \& Transcultural. Psicothema, 11, 37-51.

Sanz, J., Perdigón, A. L. \& Vázquez, C. (2003). Adaptación Española del inventario para la depresión de Beck-II (BDI-II): 2. Propiedades psicométricas en población general. Clínica \& Salud, 14, 249-280.

Sarin, S., Abela, J. R. Z. \& Auerbach, R. P. (2005). The response styles theory of depression: a test of specificity and causal mediation. Cognition and Emotion, 19, 751-761.

Sethi, S., Campbell, A. J. \& Ellis, L. A. (2010). The use of computerized self-help packages to treat adolescent depression and anxiety. Journal of Technology in Human Services, 28, 144-160.

Sheehan, D. V., Lecrubier, Y., Harnett Sheehan, K., Janavs, J., Weiller. E., Bonora, L. I., .... Dunbar, G. C. Reliability and validity of the Mini International Neuropsychiatric Interview (M. I. N. I.) according to the SCID-P. Eur Psychiatry 1997, 12, 232-241.

Slade, T., Johnston, A., Oakley-Browne, M. A., Andrews, G. \& Whiteford, H. (2009). National Survey of Mental Health and Wellbeing: methods and key findings. Australian and New Zealand Journal of Psychiatry, 43, 594-605.

Titov, N., Andrews, G., Choi, I., Schwencke, G. \& Johnston, L. (2009). Randomized controlled trial of web-based treatment of social phobia without clinician guidance. Australian and New Zealand Journal of Psychiatry, 43, 913-919.

Titov, N., Dear, B. F., Shwencke, G., Andrews, G., Johnston, L., Craske, M. G. \& McEvoy, P. (2011). Transdiagnostic internet treatment for anxiety and depression: a randomised controlled trial. Behaviour Research and Therapy, 49, 441-452.

Thompson, R. J., Mata, J., Jaeggi, S. M., Buschkuehl, M. , Jonides, J. \& Gotlib, I. H. (2010). Maladaptive coping, adaptive coping, and depressive symptoms: Variations across age and depressive state. Behaviour Research and Therapy, 1-8.

Van Straten, A., Cuijpers, P. \& Smits, N. (2008). Effectiveness of a web-based self-help intervention for symptoms of depression, anxiety, and stress: Randomized controlled trial. J Med Internet Res, 10, 1.

Vargas, B., Villamil,V., Rodríguez, C., Pérez. J. \& Cortés, J. (2011). Validación de la escala Kessler $10(\mathrm{~K}-10)$ en la detección de depresión \& ansiedad en el primer nivel de atención. Propiedades psicométricas. Salud mental, 34, 323-331.

Weissman, M. M., Bland, R. C., Canino, G. J., Faravelli, C., Green-wald, S. y Hwu, H. G., ... Yeh, E. K. (1996). Cross-national epidemiology of major depression and bipolar disorder. Jama The Journal Of The American Medical Association, 276, 293-299.

WHO Iniciative Depression in Mental Health (2010). Geneva: WHO.

Wilamowska, Z. A., Thompson-Hollands, J., Fairholme, C. P., Ellard, K. K., Farchione, T. J. \& Barlow, D. H. (2010). Conceptual background, development, and preliminary data from the unified protocol for transdiagnostic treatment of emotional disorders. Depression and Anxiety, 27, 882-890.

Wood, A., Froh, J. \& Geraghty, A. (2010). Gratitude and well-being: A review and theoretical integration. Clinical Psychology Review, 30 (7), 890-905. 